\title{
Obesity and $\mathrm{BMI}$ in myelomeningocele DB Shurtleff*, S Duguay, D Cardenas and W Walker
}

\author{
Address: Department of Pediatrics University of Washington MS: m 2-8 Seattle, WA 98195 \\ Email: DB Shurtleff* - david.shurtleff@seattlechildrens.org \\ * Corresponding author
}

from 49th Annual Meeting of the Society for Research into Hydrocephalus and Spina Bifida

Barcelona, Spain, 29 June - 2 July 2005

Published: 30 December 2005

Cerebrospinal Fluid Research 2005, 2(SuppI I):S38 doi:I0.II86/I743-8454-2-SI-S38

\section{Background}

We have analyzed 5033 visits for 663 patients to assess the most appropriate measures to determine obesity and overweight status for patients with myelomeningocele (MM) age 2-20 years of age.

\section{Materials and methods}

We recorded height or body length (Ht) (from head to hip and knee joints to sole in the presence of contracture), weight (Wt) and arm span (As) at yearly examinations $(14,828$ measures) and immediately before and after spinal fusion for 21 patients (126 measures). Ht, wt and all BMI calculations were compared by $\mathrm{T}$ tests to standards published by the National Center for Health Statistics of the US Government (NCHS). BMI was calculated with Ht and As. These two measures of BMI were also compared to each other by T tests. Multiple cell Chi Square tests were utilized to compare different levels of paralysis Lesion Level 1 (LL 1, L2 and above), LL 2 (mid-lumbar L3-5) and LL 3 (Sacral). We will show examples of Ht, Wt and DMI charts using the restricted calorie diet as recommended by Manenica (1982) demonstrating its effectiveness.

\section{Results}

Analysis of before and after scoliosis surgery height measures demonstrated the shortening effect of scoliosis on $\mathrm{Ht}$ and altered BMI calculated using Ht. $(P=<0.0001)$ Neither Wt nor Ht of patients with MM could be approximated to take into consideration lower limb hypoplasia. BMI of MM patients measured using As more closely approximated "normal" for age than BMI calculated using Ht. For example, females BMI calculated by Wt/As ${ }^{2}$ was not significantly different from NCHS data ("T" Test; $\mathrm{P}=$ 0.8 ) but calculated by $\mathrm{Wt} / \mathrm{Ht}^{2}, \mathrm{P}=0.006$ ) BMI by Chi Square comparison between younger and older male patients with MM in our series are: LL1 and LL2 patients age $2-12$ years are more obese (>95\%ile) than those age $13-20(P=0.03$ and 0.01$)$. Age has no effect on the distribution of obesity amongst LL3 males $(\mathrm{p}=0.3)$ and for female LL1, $(P=0.6)$, LL2 $(P=0.9)$, and LL3 $(P=0.2)$. BMI between different levels of lesions are insignificant between male and female LL1 $(P=1.0)$ and LL $2(P=0.9)$. LL1 $(P=0.02)$ and LL2 $(P=0.0)$ male and female patients are more frequently obese compared to LL3, $(\mathrm{P}=0.04$ and 0.007 respectively for females). Samples of these analyses will be shown.

\section{Conclusion}

Calculations of BMI using As for patients with MM are more appropriate for estimating overweight and obesity status for patients with MM than using Ht. Fewer children with $\mathrm{MM}$ in our series are overweight or obese than expected based on USA data for "normals" and published data about Spina Bifida Patients. BMI decreased following implementation of the Dietz regimen suggesting that it is an important adjunct in managing obesity. 